CARPATHIAN J. MATH.

Volume 38 (2022), No. 1,

Pages $169-178$
Online version at https : //www. carpathian. cunbm. utcluj. ro/

Print Edition: ISSN 1584 - 2851; Online Edition: ISSN 1843 - 4401

DOI: https://doi.org/10.37193/CJM.2022.01.14

Dedicated to the memory of Academician Mitrofan M. Choban (1942-2021)

\title{
Existence of common fixed points of non-linear semigroups of Enriched Kannan contractive mappings
}

\author{
SAYANTAN PANJA ${ }^{1}$, MANTU SAHA ${ }^{1}$ and RAVINDRA K. BISHT ${ }^{2}$
}

\begin{abstract}
In this article, we consider the non-linear semigroup of enriched Kannan contractive mapping and prove the existence of common fixed point on a non-empty closed convex subset $\mathcal{C}$ of a real Banach space $\mathscr{X}$, having uniformly normal structure.
\end{abstract}

\section{INTRODUCTION}

Metric fixed point theory begins with the classical Banach Contraction Principle [2] due to S. Banach in 1922. After that many famous fixed point theorems have been proved over complete metric spaces by several researchers $[4,7,15,10]$. Recently, Berinde and Păcurar [5] have introduced a new contractive mapping, called enriched Kannan mapping and proved a fixed point theorem over a Banach space, which states below:

Theorem 1.1. [5] In a Banach space $(\mathscr{X},\|\|$.$) , the enriched Kannan mapping defined as:$

$$
\|k(x-y)+\Upsilon x-\Upsilon y\| \leq \alpha(\|x-\Upsilon x\|+\|y-\Upsilon y\|) \text { for all } x, y \in \mathscr{X}
$$

for some $\alpha \in\left[0, \frac{1}{2}\right)$ and $k \in[0, \infty)$; possesses a unique fixed point in $\mathscr{X}$.

From the year 1960 onwards, common fixed point theorems for semigroups of nonlinear self-mappings play an important roles in non-linear operator theory and its applications. Several researchers have investigated various types of non-linear self-mappings, viz., uniformly Lipshitzian semigroup ([8], [23],[17]), non-expansive semigroup ([18],[19], [22]), semigroups for pseudocontractions ([9],[1]), Lipschitzian pseudocontraction semigroups [25], uniformly continuous semigroups [20] etc.

In a real Banach space $\mathscr{X}$, let $\mathcal{C} \subset \mathscr{X}$ be non-empty closed and convex. A mapping $\Upsilon: \mathcal{C} \rightarrow \mathcal{C}$ is said to be Lipschitzian mapping if for each integer $n \geq 1$ there exists positive constants $k_{n}$ such that,

$$
\left\|\Upsilon^{n} x-\Upsilon^{n} y\right\| \leq k_{n}\|x-y\| \text { for all } x, y \in \mathcal{C} .
$$

A Lipschitzian mapping is said to be $k$-uniformly Lipschitzian mapping if $k_{n} \equiv k$ for all $n \geq 1$. In the year 1973, Goebel \& Kirk [12] proved that every $k$-uniformly Lipschitzian self-mapping on a closed, convex, bounded subset $\mathcal{C}$ of a uniformly convex Banach space $\mathscr{X}$ possesses a fixed point if $k<\gamma$, where $\gamma>1$ is the unique solution of the equation $\left(1-\delta_{\mathscr{X}}\left(\frac{1}{\gamma}\right)\right) \gamma=1$; where $\delta_{\mathscr{X}}$ is the modulus of convexity of $\mathscr{X}$.

Received: 29.03.2021. In revised form: 27.10.2021. Accepted: 03.11.2021

2010 Mathematics Subject Classification. 47H10, 54H25.

Key words and phrases. Common fixed point, non-linear semigroup, enriched Kannan contractive mapping, uniformly $k$-Lipschitzian semigroup, enriched Kannan type semigroup.

Corresponding author: Ravindra K. Bisht; ravindra.bisht@yahoo.com 
In 2001, Zeng and Yang [24] proved a fixed point result of Lipschitzian semigroup, which states below.

Theorem 1.2. [24] Let $\mathcal{C}(\neq \emptyset)$ be a bounded subset of a uniformly convex Banach space $\mathscr{X}$. Let, $\mathscr{F}:\left\{\Upsilon_{s}: s \in \mathcal{G}\right\}$ be a Lipschitzian semigroup of self-mappings on $\mathcal{C}$ satisfying $\lim _{s}\left\|\Upsilon_{s}\right\|<$ $\left(\gamma_{0} \mathcal{N}(\mathscr{X})\right)^{1 / 2}$, where $\gamma_{0}=\inf \left\{\gamma: \gamma\left(1-\delta_{\mathscr{X}}(1 / \gamma)\right) \geq \frac{1}{2}\right\}$ and $\left\|\Upsilon_{s}\right\|$ is the exact Lipschitzian constant of $\Upsilon_{s}$. Moreover assume that there exists a non-empty bounded closed convex subset $\mathcal{H}$ of $\mathcal{C}$ having the following two properties:

$\left(\mathcal{P}_{1}\right) x \in \mathcal{H}$ implies that $w_{\omega}(x) \subset \mathcal{H}$, where $w_{\omega}(x)$ is the weak $\omega$-limit of $\mathscr{F}$ at $x$.

$\left(\mathcal{P}_{2}\right) \mathscr{F}$ is asymptotic regular on $\mathcal{H}$. i.e., $\lim _{t}\left\|\Upsilon_{s+t} x-\Upsilon_{t} x\right\|=0$ for all $s \in \mathcal{G}$ and $x \in \mathcal{H}$.

Then the semigroup $\mathscr{F}$ possesses a unique fixed point in $\mathcal{C}$.

Ceng et.al. [8] improved the result of Zeng and Yang [24] and proved a similar result by removing the condition of asymptotic regularity (i.e., only under the assumption of the condition $\left(\mathcal{P}_{1}\right)$ given above).

In the year 2010, Ceng et al. [8] proved the existence of common fixed point of $k$ uniformly Lipschitzian semigroup in Banach space having uniformly normal structure.

Theorem 1.3. [8] Let $\left\{\Upsilon_{s} x_{0}: s \in \mathcal{G}\right\}$ be a uniformly $k$-Lipschitzian semigroup on a non-empty closed, convex and bounded subset $\mathcal{C}$ of a real Banach space $\mathscr{X}$ with normal structure co-efficient $\mathcal{N}(\mathscr{X})>\max \left\{1, \epsilon_{0}\right\}$, where $\epsilon_{0}$ is the characteristic convexity of $\mathscr{X}$. If $\left\{\Upsilon_{s} x_{0}\right\}$ is bounded for some $x_{0} \in \mathcal{C}$, then the semigroup $\left\{\Upsilon_{s} x_{0}: s \in \mathcal{G}\right\}$ admits a common fixed point provided $k<\alpha_{*}$, where

$$
\alpha_{*}=\sup \left\{\alpha: \frac{\alpha^{2}}{\mathcal{N}(\mathscr{X})} \delta_{\mathscr{X}}^{-1}\left(1-\frac{1}{\alpha}\right) \leq 1 \text { and } 1-\frac{1}{\alpha} \in\left(0,1-\frac{\epsilon_{0}}{2}\right)\right\} .
$$

In 2017, Soliman et al.[21] introduced the uniformly generalized Kannan type semigroup and proved the existence and uniqueness theorem of common fixed point under certain condition in a Banach space.

Theorem 1.4. [21] Let $\mathcal{C}$ be a non-empty closed, convex subset of a real Banach space $\mathscr{X}$ with $\mathcal{N}(\mathscr{X})>\max \left\{1, \epsilon_{0}\right\}, \epsilon_{0}$ being the characteristic convexity of $\mathscr{X}$. Let $\left\{\Upsilon_{s}: s \in \mathcal{G}\right\}$ be a generalized uniformly Kannan type semigroup of self mapping defined on $\mathcal{C}$. i.e.,

$$
\left\|\Upsilon_{s} x-\Upsilon_{s} y\right\| \leq \alpha\left\{\left\|x-\Upsilon_{s} x\right\|+\left\|y-\Upsilon_{s} y\right\|\right\} \text { for all } x, y \in \mathcal{C}
$$

for each $s \in \mathcal{G}$ and for some $\alpha \in[0,1)$. If $\left\{\Upsilon_{s} x_{0}\right\}$ is bounded for some $x_{0} \in \mathcal{C}$, then the semigroup $\left\{\Upsilon_{s} x_{0}: s \in \mathcal{G}\right\}$ admits a common fixed point in $\mathcal{C}$, provided $\frac{3 \xi \alpha}{\mathcal{N}(\mathscr{X})} \delta_{\mathscr{X}}^{-1}\left(1-\frac{1}{\xi}\right)<1$, where $\xi=\frac{3 \alpha}{1-\alpha}$.

Very recently, in 2020, Kesahorm and Sintunavarat [16] have introduced the non-linear semigroup of weak-contraction due to Berinde and proved the existence and convergence theorem in real Banach space under certain condition, given below.

Theorem 1.5. [16] Let $\mathcal{C}$ be a non-empty closed, convex subset of a real Banach space $\mathscr{X}$ with $\mathcal{N}(\mathscr{X})>\max \left\{1, \epsilon_{0}\right\}, \epsilon_{0}$ being the characteristic convexity of $\mathscr{X}$. Let $\left\{\Upsilon_{s}: s \in \mathcal{G}\right\}$ be a weak contraction semigroup of self mappings defined on $\mathcal{C}$. i.e.,

$$
\left\|\Upsilon_{s} x-\Upsilon_{s} y\right\| \leq \alpha\|x-y\|+K\left\|y-\Upsilon_{s} x\right\| \text { for all } x, y \in \mathcal{C}
$$

for each $s \in \mathcal{G}$ and for some $\alpha \in[0,1)$ and $K \geq 0$. If $\left\{\Upsilon_{s} x_{0}: s \in \mathcal{G}\right\}$ is bounded for some $x_{0} \in \mathcal{C}$, then the semigroup $\left\{\Upsilon_{s}: s \in \mathcal{G}\right\}$ admits a common fixed point in $\mathcal{C}$, provided $K+1<\alpha_{*}$, where

$$
\alpha_{*}=\sup \left\{\beta: \frac{\beta^{2}}{\mathcal{N}(\mathscr{X})} \delta_{\mathscr{X}}^{-1}\left(1-\frac{1}{\beta}\right) \leq 1 \text { and } 1-\frac{1}{\beta} \in\left(0,1-\frac{\epsilon_{0}}{2}\right)\right\} .
$$


Inspired by this way of research, in this paper we introduce a new non-linear semigroup of enriched Kannan type contraction and prove the existence of common fixed point on a closed, convex, bounded subset of a real Banach space having uniform normal structure.

\section{PRELIMINARIES}

Let $\mathcal{C}$ be a closed, convex subset of a real Banach space $\mathscr{X}$ and $\mathcal{G}$ be an unbounded subset of $[0, \infty)$ satisfying the followings:

(i) $s+t \in \mathcal{G}$ for all $s, t \in \mathcal{G}$ (i.e., $\mathcal{G}$ is closed under addition).

(ii) $s-t \in \mathcal{G}$ for all $s, t \in \mathcal{G}$ with $s>t$.

A collection $\left\{\Upsilon_{s}: s \in \mathcal{G}\right\}$ of self-mappings defined on $\mathcal{C}$ is said to be semigroup if:

(S1) for all $s, t \in \mathcal{G}$ and $x \in \mathcal{C}, \Upsilon_{s+t} x=\Upsilon_{s}\left(\Upsilon_{t} x\right)$.

(S2) for all $x \in \mathcal{C}$, the mapping $s \mapsto \Upsilon_{s} x$ is continuous.

Chebyshev's radius and diameter of $\mathcal{C}\left(\right.$ see [11]) are denoted by $r_{\mathcal{C}}(\mathcal{C})$ and $\delta(\mathcal{C})$ respectively and defined as below:

$$
\begin{aligned}
r_{\mathcal{C}}(\mathcal{C}) & :=\inf _{x \in \mathcal{C}} \sup _{y \in \mathcal{C}}\|x-y\| \\
\delta(\mathcal{C}) & :=\sup _{x, y \in \mathcal{C}}\|x-y\| .
\end{aligned}
$$

A Banach space $\mathscr{X}$ is said to have normal structure (see [12]) if each closed, convex, bounded subset $\mathcal{C}$ (consisting more than one point) of $\mathscr{X}$, we have $r_{\mathcal{C}}(\mathcal{C})<\delta(\mathcal{C})$. If there exists a constant $k \in(0,1)$ such that $r_{\mathcal{C}}(\mathcal{C})<k \delta(\mathcal{C})$, whenever $\delta(\mathcal{C})>0$, then $\mathscr{X}$ is said to have uniform normal structure.

Normal structure co-efficient (see [6]) of a Banach space $\mathscr{X}$ is defined as:

$$
\mathcal{N}(\mathscr{X}):=\inf _{\substack{\mathcal{C} \subset \mathscr{X} \\ r_{\mathcal{C}}(\mathcal{C})>0}} \frac{\delta(\mathcal{C})}{r_{\mathcal{C}}(\mathcal{C})} .
$$

Modulus of convexity (see [13]) of a Banach space $\mathscr{X}$ is the function $\delta_{\mathscr{X}}:[0,2] \rightarrow[0,1]$ defined by

$$
\delta_{\mathscr{X}}(\epsilon):=\inf \left\{1-\left\|\frac{x+y}{2}\right\|:\|x\| \leq 1,\|y\| \leq 1 \text { and }\|x-y\| \geq \epsilon\right\}
$$

and the characteristic of convexity (see [13]) of $\mathscr{X}$ is defined as

$$
\epsilon_{0}(\mathscr{X}):=\sup \left\{\epsilon: \delta_{\mathscr{X}}(\epsilon)>0\right\} .
$$

Now we list up some properties of modulus of convexity $\delta_{\mathscr{X}}$ (see [13]):

(M1) $\delta_{\mathscr{X}}$ is monotone increasing on $[0,2]$ and strictly increasing on $\left[\epsilon_{0}, 2\right]$.

(M2) $\delta_{\mathscr{X}}$ is continuous on $[0,2)$.

(M3) $\delta_{\mathscr{X}}(0)=0$ and $\lim _{\epsilon \rightarrow 2^{-}} \delta_{\mathscr{X}}(\epsilon)=1-\frac{\epsilon_{0}}{2}$.

(M4) $\left\|a-\frac{x+y}{2}\right\| \leq r\left(1-\delta_{\mathscr{X}}\left(\frac{\epsilon}{r}\right)\right)$ whenever $\|a-x\| \leq r,\|a-y\| \leq r$ and $\|x-y\| \geq \epsilon$.

Definition 2.1. [11] For a non-empty closed, convex and bounded subset $\mathcal{C}$ of a real Banach space $\mathscr{X}$, let $\left\{x_{s}: s \in \mathcal{G}\right\}$ be a bounded net of elements of $\mathscr{X}$, where $\mathcal{G}$ is an unbounded subset of $[0, \infty)$. Then we define

- Asymptotic radius of $\left\{x_{s}: s \in \mathcal{G}\right\}$ with respect to $\mathcal{C}$ by

$$
r_{\mathcal{C}}\left(\left\{x_{s}\right\}\right):=\inf _{y \in \mathcal{C}} \limsup _{s \rightarrow \infty}\left\|x_{s}-y\right\| .
$$


- Asymptotic center of $\left\{x_{s}: s \in \mathcal{G}\right\}$ with respect to $\mathcal{C}$ by

$$
A_{\mathcal{C}}\left(\left\{x_{s}\right\}\right):=\left\{y \in \mathcal{C}: \limsup _{s \rightarrow \infty}\left\|x_{s}-y\right\|=r_{\mathcal{C}}\left(\left\{x_{s}\right\}\right)\right\} .
$$

Finally, we give the statements of the following two lemmas from [23] which are crucial in proving our main results.

Lemma 2.1. [23] Let $\mathscr{X}$ be a reflexive Banach space and $\mathcal{C}$ be a non-empty closed, convex subset of $\mathscr{X}$. Then $\mathcal{A}_{\mathcal{C}}\left(\left\{x_{t}\right\}\right)$ forms a non-empty closed, convex and bounded subset of $\mathcal{C}$, for every bounded net $\left\{x_{t}\right\}_{t \in \mathcal{G}}$ of elements of $\mathscr{X}$.

Lemma 2.2. [23] Let $\mathscr{X}$ be a Banach space having uniformly structure. Then for every bounded net $\left\{x_{t}\right\}_{t \in \mathcal{G}}$ of elements of $\mathscr{X}$, there exists $y \in \overline{c o}\left(\left\{x_{t}: t \in \mathcal{G}\right\}\right)$ such that $\limsup _{t \rightarrow \infty}\left\|x_{t}-y\right\| \leq$ $\frac{1}{\mathcal{N}(\mathscr{X})} \mathcal{D}\left(\left\{x_{t}\right\}\right)$, where $\overline{c o}(M)$ is the closure of the convex hull of $M \subset \mathscr{X}$.

\section{MAIN RESUltS}

Definition 3.2. Let $\mathcal{C}$ be a non-empty closed, convex and bounded subset of a real Banach space $\mathscr{X}$. Then the family $\left\{\Upsilon_{s}: s \in \mathcal{G}\right\}$ of self-mappings defined on $\mathcal{C}$ is said to be enriched Kannan type semigroup if the followings hold:

(a) for all $s, t \in \mathcal{G}$ and $x \in \mathcal{C}, \Upsilon_{s+t} x=\Upsilon_{s}\left(\Upsilon_{t} x\right)$.

(b) for all $x \in \mathcal{C}$, the mapping $s \mapsto \Upsilon_{s} x$ is continuous.

(c) for each $t \in \mathcal{G}, \Upsilon_{s}: \mathcal{C} \rightarrow \mathcal{C}$ satisfies

$$
\|k(x-y)+\Upsilon x-\Upsilon y\| \leq \alpha(\|x-\Upsilon x\|+\|y-\Upsilon y\|) \text { for all } x, y \in \mathcal{C}
$$

for some $\alpha \in[0,1)$ and for some $k \geq 0$.

Theorem 3.6. Let $\mathscr{X}$ be a real Banach space with normal structure coefficient $\mathcal{N}(\mathscr{X})>$ $\max \left\{1, \epsilon_{0}\right\}$, where $\epsilon_{0}$ is the characteristic convexity of $\mathscr{X}$. Let $\mathcal{C}$ be a non-empty closed, convex subset of $\mathscr{X}$ and $\mathscr{F}:=\left\{\Upsilon_{s}: s \in \mathcal{G}\right\}$ be the enriched Kannan type semigroup of self mappings defined on $\mathcal{C}$ with $0 \leq \alpha<1$ and $k \geq 0$ satisfying $\frac{k+3 \alpha}{1-\alpha}<\hat{\alpha}$, where

$$
\hat{\alpha}=\sup \left\{\beta: \frac{\beta^{2}}{\mathcal{N}(\mathscr{X})} \delta_{\mathscr{X}}^{-1}\left(1-\frac{1}{\beta}\right) \leq 1 \text { and } 1-\frac{1}{\beta} \in\left(0,1-\frac{\epsilon_{0}}{2}\right)\right\} .
$$

If $\left\{\Upsilon_{s} x_{0}: s \in \mathcal{G}\right\}$ is bounded for some $x_{0} \in \mathcal{C}$, then the semigroup $\mathscr{F}$ possesses a common fixed point in $\mathcal{C}$.

Proof. Since $\mathscr{X}$ has a uniform normal structure, it is reflexive. Since $\left\{\Upsilon_{s} x_{0}: s \in \mathcal{G}\right\}$ is bounded so by Lemma 2.1, $\mathcal{A}_{\mathcal{C}}\left(\left\{x_{0}\right\}\right)$ is a non-empty closed, convex and bounded subset of $\mathcal{C}$. Choose $x_{1} \in \mathcal{A}_{\mathcal{C}}\left(\left\{x_{0}\right\}\right)$. Then by definition of asymptotic center,

$$
\limsup _{t \rightarrow \infty}\left\|\Upsilon_{t} x_{0}-x_{1}\right\|=\inf _{y \in \mathcal{C}} \limsup _{t \rightarrow \infty}\left\|\Upsilon_{t} x_{0}-y\right\| .
$$

Since $\mathscr{F}$ is enriched Kannan type semigroup, we have

$$
\begin{aligned}
\left\|\Upsilon_{t} x_{1}\right\| & \leq\left\|\Upsilon_{t} x_{1}-\Upsilon_{t} x_{0}\right\|+\left\|\Upsilon_{t} x_{0}\right\| \\
& \leq k \cdot\left\|x_{1}-x_{0}\right\|+\alpha \cdot\left\{\left\|x_{1}-\Upsilon_{t} x_{1}\right\|+\left\|x_{0}-\Upsilon_{t} x_{0}\right\|\right\}+\left\|\Upsilon_{t} x_{0}\right\| \\
& \leq k \cdot\left\|x_{1}-x_{0}\right\|+\alpha \cdot\left\{\left\|x_{1}\right\|+\left\|\Upsilon_{t} x_{1}\right\|+\left\|x_{0}\right\|+\left\|\Upsilon_{t} x_{0}\right\|\right\}+\left\|\Upsilon_{t} x_{0}\right\|
\end{aligned}
$$

which implies $\left\|\Upsilon_{t} x_{1}\right\| \leq \frac{k}{1-\alpha}\left\|x_{1}-x_{0}\right\|+\frac{\alpha}{1-\alpha}\left\{\left\|x_{1}\right\|+\left\|x_{0}\right\|+\left\|\Upsilon_{t} x_{0}\right\|\right\}+\frac{1}{1-\alpha}\left\|\Upsilon_{t} x_{0}\right\|$. that

Thus we get, $\left\{\Upsilon_{t} x_{1}: t \in \mathcal{G}\right\}$ is bounded. Therefore we can choose $x_{2} \in \mathcal{A}_{\mathcal{C}}\left(\left\{x_{1}\right\}\right)$ such

$$
\limsup _{t \rightarrow \infty}\left\|\Upsilon_{t} x_{1}-x_{2}\right\|=\inf _{y \in \mathcal{C}} \limsup _{t \rightarrow \infty}\left\|\Upsilon_{t} x_{1}-y\right\| .
$$


Continuing this process we can construct a sequence $\left\{x_{n}\right\}$ in $\mathcal{C}$ such that,

A. for each $n \geq 0,\left\{\Upsilon_{t} x_{n}\right\}_{t \in \mathcal{G}}$ is bounded.

B. for each $n \geq 0, x_{n+1} \in \mathcal{A}_{\mathcal{C}}\left(\left\{x_{n}\right\}\right)$ such that

$\lim \sup _{t \rightarrow \infty}\left\|\Upsilon_{t} x_{n}-x_{n+1}\right\|=\inf _{y \in \mathcal{C}} \lim \sup _{t \rightarrow \infty}\left\|\Upsilon_{t} x_{n}-y\right\|=r_{\mathcal{C}}\left(\left\{\Upsilon_{t} x_{n}\right\}\right)$.

Claim 1: $\left\{x_{n}\right\}$ is a Cauchy sequence.

Let us denote $r_{\mathcal{C}}\left(\left\{\Upsilon_{t} x_{n}\right\}\right)$ simply by $r_{n}$. Then by Lemma 2.2,

$$
\begin{aligned}
r_{n} & =\limsup _{t \rightarrow \infty}\left\|\Upsilon_{t} x_{n}-x_{n+1}\right\| \\
& \leq \frac{1}{\mathcal{N}(\mathscr{X})} \mathcal{D}\left(\left\{\Upsilon_{t} x_{n}\right\}\right) \\
& =\frac{1}{\mathcal{N}(\mathscr{X})} \lim _{t \rightarrow \infty}\left(\sup _{t \leq i, j \in \mathcal{G}}\left\|\Upsilon_{i} x_{n}-\Upsilon_{j} x_{n}\right\|\right) \\
& =\frac{1}{\mathcal{N}(\mathscr{X})} \lim _{t \rightarrow \infty}\left(\sup _{t \leq i, j \in \mathcal{G}}\left\|\Upsilon_{i} x_{n}-\Upsilon_{i} \Upsilon_{j-i} x_{n}\right\|\right) \\
& \leq \frac{1}{\mathcal{N}(\mathscr{X})} \lim _{t \rightarrow \infty}\left(\sup _{t \leq i, j \in \mathcal{G}}\left\{k \cdot\left\|x_{n}-\Upsilon_{j-i} x_{n}\right\|+\alpha\left\|x_{n}-\Upsilon_{i} x_{n}\right\|+\alpha\left\|\Upsilon_{j} x_{n}-\Upsilon_{j-i} x_{n}\right\|\right\}\right) \\
& \leq \frac{1}{\mathcal{N}(\mathscr{X})}\left[k \cdot d\left(x_{n}\right)+\alpha\left\{d\left(x_{n}\right)+d\left(x_{n}\right)+d\left(x_{n}\right)\right\}\right] \\
& =\frac{1}{\mathcal{N}(\mathscr{X})}(k+3 \alpha) d\left(x_{n}\right)
\end{aligned}
$$

where $d\left(x_{n}\right)=\sup _{t \in \mathcal{G}}\left\|x_{n}-\Upsilon_{t} x_{n}\right\|$.

Now if $d\left(x_{n}\right)=0$ for some $n \in \mathbb{N}$, then $x_{n}$ is a common fixed point of the semigroup $\mathscr{F}$ and hence we are done. So suppose, $d\left(x_{n}\right)>0$, for all $n \geq 0$. Let $\epsilon>0$ be arbitrary and $n \geq 0$ be fixed.

Since $d\left(x_{n+1}\right)=\sup _{t \in \mathcal{G}}\left\|\Upsilon_{t} x_{n+1}-x_{n+1}\right\|$, by definition of supremum, there exists $j \in \mathcal{G}$ such that

$$
\left\|\Upsilon_{j} x_{n+1}-x_{n+1}\right\|>d\left(x_{n+1}\right)-\epsilon .
$$

Since $r_{n}=\limsup _{t \rightarrow \infty}\left\|T_{t} x_{n}-x_{n+1}\right\|$, so by definition of limit superior, there exists $h \in \mathcal{G}$ such that

So for all $s \geq h$,

$$
\sup _{s \geq h}\left\|\Upsilon_{s} x_{n}-x_{n+1}\right\|<r_{n}+\epsilon
$$

$$
\left\|\Upsilon_{s} x_{n}-x_{n+1}\right\|<r_{n}+\epsilon
$$

Now for $s \geq h+j$,

$$
\begin{aligned}
\left\|\Upsilon_{s} x_{n}-\Upsilon_{j} x_{n+1}\right\| & =\left\|\Upsilon_{j+(s-j)} x_{n}-\Upsilon_{j} x_{n+1}\right\| \\
& \leq k .\left\|\Upsilon_{s-j} x_{n}-x_{n+1}\right\|+\alpha \cdot\left\{\left\|\Upsilon_{s} x_{n}-\Upsilon_{s-j} x_{n}\right\|+\left\|x_{n+1}-\Upsilon_{j} x_{n+1}\right\|\right\} \\
& <(k+3 \alpha) \cdot\left(r_{n}+\epsilon\right)+\alpha \cdot\left\|\Upsilon_{s} x_{n}-\Upsilon_{j} x_{n+1}\right\|
\end{aligned}
$$

implying that

$$
\left\|\Upsilon_{s} x_{n}-\Upsilon_{j} x_{n+1}\right\|<\gamma\left(r_{n}+\epsilon\right), \text { where } \gamma=\frac{k+3 \alpha}{1-\alpha} .
$$

Now using (3.6), (3.7), (3.8) and property (M4) we have for $s \geq h+j$,

$$
\left\|\Upsilon_{s} x_{n}-\frac{1}{2}\left(x_{n+1}+\Upsilon_{j} x_{n+1}\right)\right\| \leq \gamma\left(r_{n}+\epsilon\right)\left(1-\delta_{\mathscr{X}}\left(\frac{d\left(x_{n+1}\right)-\epsilon}{\gamma\left(r_{n}+\epsilon\right)}\right)\right) .
$$

Therefore,

$$
\begin{aligned}
r_{n} & \leq \limsup _{s \rightarrow \infty}\left\|\Upsilon_{s} x_{n}-\frac{1}{2}\left(x_{n+1}+\Upsilon_{j} x_{n+1}\right)\right\| \\
& \leq \gamma\left(r_{n}+\epsilon\right)\left(1-\delta_{\mathscr{X}}\left(\frac{d\left(x_{n+1}\right)-\epsilon}{\gamma\left(r_{n}+\epsilon\right)}\right)\right) .
\end{aligned}
$$


Letting $\epsilon \rightarrow 0$ we get, $r_{n} \leq \gamma r_{n}\left(1-\delta_{\mathscr{X}}\left(\frac{d\left(x_{n+1}\right)}{\gamma r_{n}}\right)\right)$ which yields,

$$
\delta_{\mathscr{X}}\left(\frac{d\left(x_{n+1}\right)}{\gamma r_{n}}\right) \leq 1-\frac{1}{\gamma} .
$$

Claim 2: $d\left(x_{n+1}\right) \leq \gamma r_{n} \delta_{\mathscr{X}}^{-1}\left(1-\frac{1}{\gamma}\right)$.

If $\frac{d\left(x_{n+1}\right)}{\gamma r_{n}} \in\left[\epsilon_{0}, 2\right)$, then as $\delta_{\mathscr{X}}^{-1}$ exists so, $\frac{d\left(x_{n+1}\right)}{\gamma r_{n}} \leq \delta_{\mathscr{X}}^{-1}\left(1-\frac{1}{\gamma}\right)$ and we are done.

Now consider, $\frac{d\left(x_{n+1}\right)}{\gamma r_{n}} \in\left[0, \epsilon_{0}\right)$. Since $\delta_{\mathscr{X}}:\left[\epsilon_{0}, 2\right) \rightarrow \delta_{\mathscr{X}}\left(\left[\epsilon_{0}, 2\right)\right)=\left[0,1-\frac{\epsilon_{0}}{2}\right)$ is a bijection and $1-\frac{1}{\gamma} \in\left[0,1-\frac{\epsilon_{0}}{2}\right)$, from the hypothesis $\gamma=\frac{k+3 \alpha}{1-\alpha}<\hat{\alpha}$, we get $\delta_{\mathscr{X}}^{-1}\left(1-\frac{1}{\gamma}\right) \geq \epsilon_{0}$, which implies that $\frac{d\left(x_{n+1}\right)}{\gamma r_{n}} \leq \delta_{\mathscr{X}}^{-1}\left(1-\frac{1}{\gamma}\right)$. Therefore we have,

$$
\begin{aligned}
d\left(x_{n+1}\right) & \leq \gamma r_{n} \delta_{\mathscr{X}}^{-1}\left(1-\frac{1}{\gamma}\right)(\text { Claim } 2 \text { is justified }) \\
& \leq \frac{\gamma}{\mathcal{N}(\mathscr{X})}(k+3 \alpha) d\left(x_{n}\right) \delta_{\mathscr{X}}^{-1}\left(1-\frac{1}{\gamma}\right) \\
& =\mathcal{A} d\left(x_{n}\right)
\end{aligned}
$$

where, $\mathcal{A}=\frac{\gamma}{\mathcal{N}(\mathscr{X})}(k+3 \alpha) \delta_{\mathscr{X}}^{-1}\left(1-\frac{1}{\gamma}\right)<\frac{\gamma^{2}}{\mathcal{N}(\mathscr{X})} \delta_{\mathscr{X}}^{-1}\left(1-\frac{1}{\gamma}\right)<1$.

Then by induction,

$$
d\left(x_{n}\right) \leq \mathcal{A} d\left(x_{n-1}\right) \leq \mathcal{A}^{2} d\left(x_{n-2}\right) \leq \cdots \leq \mathcal{A}^{n} d\left(x_{0}\right) .
$$

Now for each $n \in \mathbb{N}$,

$$
\begin{aligned}
\left\|x_{n+1}-x_{n}\right\| & \leq \limsup _{t \rightarrow \infty}\left\|\Upsilon_{t} x_{n}-x_{n+1}\right\|+\limsup _{t \rightarrow \infty}\left\|\Upsilon_{t} x_{n}-x_{n}\right\| \\
& \leq r_{n}+d\left(x_{n}\right) \\
& \leq\left(\frac{k+3 \alpha}{\mathcal{N}(\mathscr{X})}+1\right) \mathcal{A}^{n} d\left(x_{0}\right) .
\end{aligned}
$$

Since $\mathcal{A}<1$, so $\sum_{n=1}^{\infty}\left\|x_{n+1}-x_{n}\right\|$ is convergent and consequently $\left\{x_{n}\right\}$ is a Cauchy sequence. Thus Claim 1 is justified.

By completeness of $\mathcal{C}$, sequence $\left\{x_{n}\right\}$ is convergent and let $x_{n} \rightarrow x_{*} \in \mathcal{C}$ as $n \rightarrow \infty$. Finally for each $t \in \mathcal{G}$ we have,

$$
\begin{aligned}
\left\|x_{*}-\Upsilon_{t} x_{*}\right\| & \leq\left\|x_{*}-x_{n}\right\|+\left\|x_{n}-\Upsilon_{t} x_{n}\right\|+\left\|\Upsilon_{t} x_{n}-\Upsilon_{t} x_{*}\right\| \\
& \leq\left\|x_{*}-x_{n}\right\|+\left\|x_{n}-\Upsilon_{t} x_{n}\right\|+k\left\|x_{n}-x_{*}\right\|+\alpha\left(\left\|x_{n}-\Upsilon_{t} x_{n}\right\|+\left\|x_{*}-\Upsilon_{t} x_{*}\right\|\right)
\end{aligned}
$$

which yields,

$$
(1-\alpha)\left\|x_{*}-\Upsilon_{t} x_{*}\right\| \leq(1+k)\left\|x_{n}-x_{*}\right\|+(1+\alpha) d\left(x_{n}\right) \rightarrow 0 \text { as } n \rightarrow \infty .
$$

Consequently, $\Upsilon_{t} x_{*}=x_{*}$ for all $t \in \mathcal{G}$.

Now we are going to present an example corresponding to the Theorem 3.6. Before that, we note down some results which are needed to establish the example.

- (See [14]) For the space $L^{p}$ with $p \geq 2$, we have the modulus of convexity $\delta_{\mathscr{X}}(\epsilon)=$ $1-\left(1-\left(\frac{\epsilon}{2}\right)^{p}\right)^{1 / p}$.

- (See [12]) If $\delta_{\mathscr{X}}(1)>0$ (i.e., if $\epsilon_{0}(\mathscr{X})<1$ ), then $\mathscr{X}$ has normal structure. 
- (See [3]) If $\Omega$ is a $\sigma$-finite measure space, then the normal structure co-efficient of the space $L^{p}(\Omega), 1 \leq p<\infty$ is given by $\mathcal{N}\left(L^{p}(\Omega)\right)=\min \left\{2^{1-1 / p}, 2^{1 / p}\right\}$.

Example 3.1. Consider, $\mathscr{X}=L^{2}([-10,10])$ and let $\mathcal{C}=\mathscr{X}$. Then $\mathscr{X}$ is a real Banach space. Since $[-10,10]$ is a $\sigma$-finite measured space, so $\delta_{\mathscr{X}}(1)=1-\frac{\sqrt{3}}{4}>0$. Then $\epsilon_{0}(\mathscr{X})<1$ and $\mathscr{X}$ has normal structure with normal structure co-efficient $\mathcal{N}(\mathscr{X})=\min \left\{2^{1-\frac{1}{2}}, 2^{\frac{1}{2}}\right\}=$ $\sqrt{2}>\max \left\{1, \epsilon_{0}\right\}$.

Now,

$$
\begin{aligned}
\hat{\alpha} & =\sup \left\{\beta: \frac{\beta^{2}}{\mathcal{N}(\mathscr{X})} \delta_{\mathscr{X}}^{-1}\left(1-\frac{1}{\beta}\right) \leq 1 \text { and } 1-\frac{1}{\beta} \in\left(0,1-\frac{\epsilon_{0}}{2}\right)\right\} \\
& =\sup \left\{\beta: \delta_{\mathscr{X}}\left(\frac{\sqrt{2}}{\beta^{2}}\right) \geq 1-\frac{1}{\beta} \text { and } 1-\frac{1}{\beta} \in\left(0,1-\frac{\epsilon_{0}}{2}\right)\right\} .
\end{aligned}
$$

By doing some elementary calculation we get, $\hat{\alpha}=\left(\frac{\sqrt{3}}{2}+\frac{1}{2}\right)^{1 / 2}$.

Now we consider the collection of mappings $\mathscr{F}:=\left\{\Upsilon_{s}: \mathcal{C} \rightarrow \mathcal{C} \mid s \in \mathcal{G}\right\}$ such that

$$
\left(\Upsilon_{s} f\right)(x)=5^{-\frac{s}{3}} f(x) \text { for all } f \in \mathcal{C},
$$

where $\mathcal{G}:=\{5 n: n \in \mathbb{N}\}$, an unbounded subset of $[0, \infty)$ satisfying $s+t \in \mathcal{G}$ for all $s, t \in \mathcal{G}$ and $s-t \in \mathcal{G}$ for all $s, t \in \mathcal{G}$ with $s>t$.

It is clearly seen that the collection $\mathscr{F}$ satisfies the property (S2). Again for all $s, t \in \mathcal{G}$ and for all $f \in \mathcal{C}$ we have, $\Upsilon_{s+t} f=5^{-\frac{s+t}{3}} f=5^{-\frac{s}{3}}\left(5^{-\frac{t}{3}} f\right)=5^{-\frac{s}{3}}\left(\Upsilon_{t} f\right)=\Upsilon_{s}\left(\Upsilon_{t} f\right)$. Thus property (S1) is satisfied.

Moreover, the function $s \mapsto 5^{-s / 3}$ is bounded measurable function on $[-10,10]$ and hence the collection $\mathscr{F}$ forms a semigroup of self mappings defined on $\mathcal{C}$. Now we will verify that $\Upsilon_{s}$ is the enriched Kannan type mapping with $\alpha=\frac{9}{40}$ and $k=\frac{1}{10}$.

For all $f, g \in \mathcal{C}$ and for all $s \in \mathcal{G}$ we have,

$$
\left\|k(f-g)+\Upsilon_{s} f-\Upsilon_{s} g\right\|=\left\|k(f-g)+5^{-\frac{s}{3}} f-5^{-\frac{s}{3}} g\right\|=\left(\frac{1}{10}+5^{-\frac{s}{3}}\right)\|f-g\|
$$

and

$$
\begin{aligned}
\alpha\left(\left\|f-\Upsilon_{s} f\right\|+\left\|g-\Upsilon_{s} g\right\|\right) & =\frac{9}{40}\left(\left\|f-5^{-\frac{s}{3}} f\right\|+\left\|g-5^{-\frac{s}{3}} g\right\|\right) \\
& =\frac{9}{40}\left(1-5^{-\frac{s}{3}}\right)(\|f\|+\|g\|) .
\end{aligned}
$$

Now by the triangle inequality, $\|f-g\| \leq\|f\|+\|g\|$ and it is easy to see that for all $s \in \mathcal{G},\left(\frac{1}{10}+5^{-\frac{s}{3}}\right) \leq \frac{9}{40}\left(1-5^{-\frac{s}{3}}\right)$.

Thus from (3.13) and (3.14), it follows that,

$$
\left\|k(f-g)+\Upsilon_{s} f-\Upsilon_{s} g\right\| \leq \alpha\left(\left\|f-\Upsilon_{s} f\right\|+\left\|g-\Upsilon_{s} g\right\|\right) .
$$

Therefore the family $\mathscr{F}=\left\{\Upsilon_{s}: s \in \mathcal{G}\right\}$ forms the enriched Kannan type semigroup of self mappings defined on $\mathcal{C}$ with $\alpha=\frac{9}{40}, k=\frac{1}{10}$ and then $\frac{k+3 \alpha}{1-\alpha}=1<\left(\frac{\sqrt{3}}{2}+\frac{1}{2}\right)^{1 / 2}=\hat{\alpha}$. Moreover, $\left\{\Upsilon_{s} 0_{\text {function }}: s \in \mathcal{G}\right\}=\left\{0_{\text {function }}\right\}$, which is bounded trivially.

Thus all the conditions of Theorem 3.6 are satisfied and it is seen that $f \equiv 0_{\text {function }} \in \mathcal{C}$ is the common fixed point of the semigroup $\mathscr{F}$.

Corollary 3.1. If $k=0$, then the enriched Kannan type mapping (3.5) reduces to the generalized uniformly Kannan mapping (1.3) and Theorem 3.6 generalizes Theorem 1.4.

Corollary 3.2. If $\alpha=0$, then the enriched Kannan type mapping (3.5) reduces to the uniformly $k$-Lipschitzian mapping (1.2) and Theorem 3.6 generalizes Theorem 1.3. 
Theorem 3.7. Let $\mathcal{C}(\neq \emptyset)$ be a bounded subset of a uniformly convex Banach space $\mathscr{X}$. Let $\mathscr{F}:=\left\{\Upsilon_{s}: s \in \mathcal{G}\right\}$ be the enriched Kannan type semigroup of self mappings defined on $\mathcal{C}$ satisfying $\frac{k+3 \alpha}{1-\alpha}<\mu_{0} \mathcal{N}(\mathscr{X})$, where $\mu_{0}=\inf \left\{\mu \geq 1: \mu\left(1-\delta_{\mathscr{X}}\left(\frac{1}{\mu}\right)\right) \geq \frac{1}{2}\right\}$. Also suppose that there exists a non-empty, closed, convex and bounded subset $\mathcal{H}$ of $\mathcal{C}$ satisfying the following property:

$(\mathscr{P}) x \in \mathcal{H}$ implies $w_{\omega}(x) \subset \mathcal{H}$ where, $w_{\omega}(x):=\left\{y \in \mathscr{X}: y=\right.$ weak $-\lim _{s_{\alpha}} \Upsilon_{s_{\alpha}} x$ for some subnet $\left.\left\{s_{\alpha}\right\} \subset \mathcal{G}\right\}$.

Then there exists $x_{*} \in \mathcal{H}$ such that $\Upsilon_{s} x_{*}=x_{*}$.

Proof. Let $x_{0} \in \mathcal{H}$. For each $t \in \mathcal{G}$ consider the bounded net $\left\{\Upsilon_{s} x_{0}: t \leq s \in \mathcal{G}\right\}$. From Lemma 2.2, there exists $y_{t} \in \overline{c o}\left\{\Upsilon_{s} x_{0}: t \leq s \in \mathcal{G}\right\}$ such that

$$
\limsup _{s \rightarrow \infty}\left\|\Upsilon_{s} x_{0}-y_{t}\right\| \leq \frac{1}{\mathcal{N}(\mathscr{X})} \mathcal{D}\left(\left\{\Upsilon_{s} x_{0}: t \leq s \in \mathcal{G}\right\}\right) .
$$

Since $\mathscr{X}$ is reflexive, $\left\{y_{t}\right\}$ has a subnet $\left\{y_{t_{l}}\right\}$ which converges weakly to some $x_{1}$ for some $x_{1} \in \mathscr{X}$. From (3.16) and due to weakly lower semi-continuity of the functional $\lim \sup _{t \rightarrow \infty}\left\|\Upsilon_{t} x_{0}-y\right\|$, we obtain

$$
\limsup _{t \rightarrow \infty}\left\|\Upsilon_{t} x_{0}-x_{1}\right\| \leq \frac{1}{\mathcal{N}(\mathscr{X})} \mathcal{D}\left(\left\{\Upsilon_{s} x_{0}: t \leq s \in \mathcal{G}\right\}\right)
$$

Also, it can be easily seen that $x_{1} \in \cap_{t \in \mathcal{G}} \overline{c o}\left\{\Upsilon_{s} x_{0}: t \leq s \in \mathcal{G}\right\}$ such that for all $z \in \mathscr{X}$,

$$
\left\|z-x_{1}\right\| \leq \limsup _{t \rightarrow \infty}\left\|z-\Upsilon_{t} x_{0}\right\| .
$$

Now, using the property $(\mathscr{P})$ and the fact that $\cap_{t \in \mathcal{G}} \overline{c o}\left\{\Upsilon_{s} x_{0}: t \leq s \in \mathcal{G}\right\}=\overline{c o}\left\{w_{w}\left(x_{0}\right)\right\}$ we can conclude that $x_{1} \in \mathcal{H}$. On repeating this process we obtain a sequence $\left\{x_{n}\right\}$ in $\mathcal{H}$ such that

$$
\text { a. } \limsup _{t \rightarrow \infty}\left\|\Upsilon_{t} x_{n}-x_{n+1}\right\| \leq \frac{1}{\mathcal{N}(\mathscr{X})} \mathcal{D}\left(\left\{\Upsilon_{t} x_{n}: t \in \mathcal{G}\right\}\right)
$$

and

$$
\text { b. }\left\|z-x_{n+1}\right\| \leq \limsup _{t \rightarrow \infty}\left\|z-\Upsilon_{t} x_{n}\right\| \text {. }
$$

Letting $r_{n}:=\lim \sup _{t \rightarrow \infty}\left\|\Upsilon_{t} x_{n}-x_{n+1}\right\|$, along the same line of proof as in Theorem 3.6 we can get

$$
r_{n} \leq \frac{1}{\mathcal{N}(\mathscr{X})}(k+3 \alpha) d\left(x_{n}\right) \text { for all } n=0,1,2, \cdots
$$

and for all $s \geq h+j$,

$$
\left\|\Upsilon_{s} x_{n}-\frac{1}{2}\left(x_{n+1}+\Upsilon_{j} x_{n+1}\right)\right\| \leq \gamma\left(r_{n}+\epsilon\right)\left(1-\delta_{\mathscr{X}}\left(\frac{d\left(x_{n+1}\right)-\epsilon}{\gamma\left(r_{n}+\epsilon\right)}\right)\right)
$$

where, $\gamma=\frac{k+3 \alpha}{1-\alpha}$.

For $z=\frac{1}{2}\left(x_{n+1}+\Upsilon_{j} x_{n+1}\right) \in \mathscr{X}$ we have from equation (3.20),

$$
\begin{aligned}
\frac{1}{2}\left(d\left(x_{n+1}\right)-\epsilon\right) & <\left\|\frac{1}{2}\left(x_{n+1}+\Upsilon_{j} x_{n+1}\right)\right\| \\
& \leq \limsup _{t \rightarrow \infty}\left\|\Upsilon_{t} x_{n}-\frac{1}{2}\left(x_{n+1}+\Upsilon_{j} x_{n+1}\right)\right\| \\
& \leq \gamma\left(r_{n}+\epsilon\right)\left(1-\delta \mathscr{X}\left(\frac{d\left(x_{n+1}\right)-\epsilon}{\gamma\left(r_{n}+\epsilon\right)}\right)\right) .
\end{aligned}
$$

Passing the limit $\epsilon \rightarrow 0$ we get,

$$
\frac{1}{2} d\left(x_{n+1}\right) \leq \gamma r_{n}\left(1-\delta_{\mathscr{X}}\left(\frac{d\left(x_{n+1}\right)}{\gamma r_{n}}\right)\right) .
$$


Again from equation (3.18), it can be easily seen that for each $j \in \mathcal{G}$,

$$
\left\|\Upsilon_{j} x_{n+1}-x_{n+1}\right\| \leq \limsup _{t \rightarrow \infty}\left\|\Upsilon_{j} x_{n+1}-\Upsilon_{t} x_{n+1}\right\| \leq \gamma r_{n}
$$

which yields

$$
d\left(x_{n+1}\right) \leq \gamma r_{n}
$$

Now combining equations (3.22) and (3.23) and using the definition of $\mu_{0}$, we derive that $\frac{\gamma r_{n}}{d\left(x_{n+1}\right)} \geq \mu_{0}$. Hence from (3.21),

$$
d\left(x_{n+1}\right) \leq \frac{\gamma}{\mu_{0}} r_{n} \leq \frac{\gamma^{2}}{\mu_{0} \mathcal{N}(\mathscr{X})} d\left(x_{n}\right) .
$$

Setting $\mathcal{A}=\frac{\gamma^{2}}{\mu_{0} \mathcal{N}(\mathscr{X})}<1$ (by assumption) we deduce that

$$
d\left(x_{n}\right) \leq \mathcal{A d}\left(x_{n-1}\right) \leq \cdots \leq \mathcal{A}^{n} d\left(x_{0}\right)
$$

and from the fact that $\left\|x_{n+1}-x_{n}\right\| \leq\left(\frac{k+3 \alpha}{\mathcal{N}(\mathscr{X})}+1\right) \mathcal{A}^{n} d\left(x_{0}\right)$ [see (3.12)] which implies that $\sum_{n=1}^{\infty}\left\|x_{n+1}-x_{n}\right\|$ is convergent which in turns $\left\{x_{n}\right\}$ converges strongly and let $\lim _{n \rightarrow \infty} x_{n}=x_{*}$, for some $x_{*} \in \mathcal{H}$. Now for each $t \in \mathcal{G}$ we have,

$$
\begin{aligned}
\left\|x_{*}-\Upsilon_{t} x_{*}\right\| & \leq\left\|x_{*}-x_{n}\right\|+\left\|x_{n}-\Upsilon_{t} x_{n}\right\|+\left\|\Upsilon_{t} x_{n}-\Upsilon_{t} x_{*}\right\| \\
& \leq\left\|x_{*}-x_{n}\right\|+\left\|x_{n}-\Upsilon_{t} x_{n}\right\|+k\left\|x_{n}-x_{*}\right\|+\alpha\left(\left\|x_{n}-\Upsilon_{t} x_{n}\right\|+\left\|x_{*}-\Upsilon_{t} x_{*}\right\|\right)
\end{aligned}
$$

which yields,

$$
(1-\alpha)\left\|x_{*}-\Upsilon_{t} x_{*}\right\| \leq(1+k)\left\|x_{n}-x_{*}\right\|+(1+\alpha) d\left(x_{n}\right) \rightarrow 0 \text { as } n \rightarrow \infty .
$$

Consequently, $\Upsilon_{t} x_{*}=x_{*}$ for all $t \in \mathcal{G}$.

Example 3.2. Considering the example as in Example 3.1, and letting $\mathcal{H}=\mathcal{C}$, we have $\mathscr{X}$ is the enriched Kannan type semigroup defined on $\mathcal{C}$.

Now,

$$
\begin{aligned}
\mu_{0} & =\inf \left\{\mu \geq 1: \mu\left(1-\delta_{\mathscr{X}}\left(\frac{1}{\mu}\right)\right) \geq \frac{1}{2}\right\} \\
& =\inf \left\{\mu \geq 1: \mu\left(1-\frac{1}{4 \mu^{2}}\right)^{1 / 2} \geq \frac{1}{2}\right\} \\
& =1 .
\end{aligned}
$$

Then, $\frac{k+3 \alpha}{1-\alpha}=1<\sqrt{2}=\mu_{0} \mathcal{N}(\mathscr{X})$. Also it is easy to see that, weak $\omega$-limit of $\mathscr{F}$ at $f$ is $w_{\omega}(f)=\left\{0_{\text {function }}\right\} \subset \mathcal{H}$. Thus all the conditions of Theorem 3.7 are satisfied and $0_{\text {function }}$ is the common fixed point of the semigroup $\mathscr{F}$.

Conclusion. In this paper, we consider the non-linear semigroup of enriched Kannan type contractive mappings over real Banach spaces and prove the existence of common fixed point of this semigroup under certain assumptions on the underlying space and the mappings. Examples are given to illustrate the feasibility of our fixed point theorems. In future, one can find several results in this connection by considering different types of contractive and non-expansive mappings like enriched Chatterjea type mappings. Also one can try to prove our theorems by using some weaker conditions.

Acknowledgments. The authors would like to thank both the eminent reviewers for their valuable suggestions on the manuscript which helped us to improve our original draft of this paper. The first author gratefully acknowledges financial support awarded by the Council of Scientific and Industrial Research (CSIR), New Delhi, India, through research fellowship for carrying out research work leading to the preparation of this manuscript. 


\section{REFERENCES}

[1] Agarwal, R. P.; Qin, X.; Kang, S. M. Strong convergence theorems for strongly continuous semigroups of pseudocontractions. Appl. Math. Lett. 24 (2011), 1845-1848.

[2] Banach, S., Sur les operations dans les ensembles abstraits et leur application aux equations untegrales. Fund. Math. 3 (1922), 133-181.

[3] Benavides, T. Normal structure coefficients of $L^{p}(\Omega)$, Proceedings of the Royal Society of Edinburgh: Section A Mathematics. 117 (1991), no. 3-4, 299-303.

[4] Berinde, V., Approximating fixed points of weak contractions using the Picard iteration. Nonlinear Anal. Forum 9 (2004), no. 1, 43-53.

[5] Berinde, V.; Păcurar, M. Kannan's fixed point approximation for solving split feasibility and variational inequality problems. J. Comput. Appl. Math. 386 (2021), 113217, 9 pp.

[6] Bynum, W. L. Normal structure coefficients for Banach spaces. Pac. J. Math. 86 (1980), 427-436.

[7] Chatterjea, S. K. Fixed-point theorems. C. R. Acad. Bulgare Sci. 25 (1972), 727-730.

[8] Ceng, L. C.; Xu, H. K. Yao, J. C. Uniformly normal structure and uniformly Lipschitzian semigroups. Nonlinear Anal. 73 (2010), 3742-3750.

[9] Cho, S. Y.; Kang, S. M. Approximation of fixed points of pseudocontraction semigroups based on viscosity iteration process. Appl. Math. Lett. 24 (2011), 224-228.

[10] Ćirić, L. B. A generalization of Banach's contraction principle. Proc. Amer. Math. Soc. 45 (1974), no. 2, 267-273.

[11] Edelstein, M. The construction of an asymptotic center with a fixed-point property. Bull. Am. Math. Soc. 78 (1972), 206-208.

[12] Goebel, K.; Kirk, W. A. A fixed point theorem for transformations whose iterates have uniform Lipschitz constant. Stud. Math. 47 (1973), 135-140.

[13] Goebel, K.; Reich, S. Uniform convexity, hyperbolic geometry and nonexpansive mappings, Pure and Applied Mathematics, A Series of Monograph and Textbooks, vol. 83, Marcel Dekker, New York (1984).

[14] Hanner, O. On the uniform convexity of $L^{p}$ and $l^{p}$. Arkiv för Matematik 3 (1956), no. 3, 239-244.

[15] Kannan, R. Some Results on Fixed Points. Bull. Cal. Math. Soc. 60 (1968), 71-76.

[16] Kesahorm, T.; Sintunavarat, W. Existence and convergence theorems concerning common fixed points of nonlinear semigroups of weak contractions. J. Fixed Point Theory Appl. 22 (2020), 70, 19 pp.

[17] Kozlowski, W. M. Monotone Lipschitzian semigroups in Banach spaces. J. Aust. Math. Soc. 105 (2018), 1-12.

[18] Lau, Anthony To-Ming; Zhang, Yong. Fixed point properties of semigroups of non-expansive mappings. J. Funct. Anal. 254 (2008), no. 10, 2534-2554.

[19] Li, S.; Li, L.; Su, Y. General iterative methods for one-parameter nonexpansive semigroup in Hilbert space. Nonlinear Anal. 70 (2009), 3065-3071.

[20] Soliman, A. H. A common fixed point theorem for semigroups of non-linear uniformly continuous mappings with an application to asymptotic stability of non-linear system. Filomat 31 (2017), no. 7, 1949-1957.

[21] Soliman, A. H.; Imdad, M.; Ahmadullah, M. Fixed point theorems for generalized Kannan type semigroup of self-mappings. Creat. Math. Inform. 26 (2017), No. 2, 231-240.

[22] Suzuki, T. On strong convergence to common fixed points of nonexpansive semigroups in Hilbert spaces. Proc. Am. Math. Soc. 131 (2003), 2133-2136.

[23] Tan, K. K.; Xu, H. K. Fixed point theorems for Lipschitzian semigroups in Banach spaces. Nonlinear Anal. 20 (1993), 395-404.

[24] Zeng L. C.; Yang Y. L. On the existence of fixed points for Lipschitzian semigroups in Banach spaces. Chinese Ann. Math. 22B (2001), no. 3, 397-404.

[25] Zhang, S. Convergence theorem of common fixed points for Lipschitzian pseudo-contraction semigroups in Banach spaces. Appl. Math. Mech. Engl. Ed. 30 (2009), 145-152.

${ }^{1}$ Department of Mathematics

THE UNIVERSITY OF BURDWAN

Purba BARDHAMAN-713104, West BENGAL, INDIA

Email address: spanja1729@gmail.com

Email address: mantusaha. bu@gmai l. com

${ }^{2}$ Department of Mathematics

National Defence ACADEMY

KHADAKWASLA-411023, PUNE, INDIA

Email address: ravindra.bisht@yahoo.com 